\title{
Impact of L-DOPA treatment of patients with Parkinson's disease on mononuclear subsets and phagocytosis in the peripheral blood
}

\author{
Artur Hurny', Grażyna Michałowska-Wender2,3, Mieczysław Wender ${ }^{3}$ \\ ${ }^{1}$ Regional Hospital for Neurological and Psychiatric Diseases, Cibórz, Poland, 2Laboratory of Neurogenetics, Department of Neurology, \\ University of Medical Sciences, Poznań, Poland, ${ }^{3}$ Neuroimmunological Unit, Mossakowski Medical Research Centre, Polish Academy \\ of Sciences, Poznań, Poland
}

\begin{abstract}
Until now, the question as to the role of immunological mechanisms in neuronal death of extrapyramidal cell systems in Parkinson's disease (PD) has not been fully resolved.

One of the approaches includes examination of circulating blood cells. In our studies on 24 PD patients, peripheral blood was studied before and after medication with L-DOPA compounds. Patients with PD demonstrated an increase in lymphocyte CD95/CD3 ratio as well as a considerable number of cells dead due to apoptotic processes. After treatment with L-DOPA, both the CD95/CD3 ratio, assumed to represent an antigen marker characteristic of apoptotic cells, and the number of cells dead due to apoptotic processes were found to be decreased. Thus, the findings indicate that levodopa treatment in PD has an impact on apoptotic processes and this should be taken into consideration as a positive event in the pathomechanism precipitated by this treatment.
\end{abstract}

Key words: L-DOPA, Parkinson's disease, mononuclear subset, apoptosis, phagocytosis.

\section{Introduction}

Parkinson's disease (PD) is a neurodegenerative disorder of middle- and old-aged humans. The overwhelming neurochemical dysfunction involves loss of dopamine in nigrostriatal and in other neurotransmitter systems, including noradrenergic and cholinergic ones, accompanied by persistent equilibrium in the immune system [1]. Numerous reports, starting with the important paper of McGeer et al. [11], have indicated that an inflammatory process in the brain in the form of microglial activation, acts as a factor which triggers the degenerative process. However, it is not clear whether the microglial activation is the cause of cellular lesions and cell death or it is only a secondary reaction, developing in the course of neuronal degeneration in the brain [12].

An impact of immune factors on the pathomechanism of PD has been discussed for years $[4-9,13,14]$.

The basic, but so far unresolved question involves the role of immunological mechanisms in neuronal death within the extrapyramidal cell system. One possible approach in respective studies involves examination of circulating blood cells and humoral cytokines in PD patients. The problem is not new, but so far 
the question whether medication with L-DOPA compounds has any important impact on immunological events in peripheral blood of PD patients remains unresolved. Due to its therapeutic implications the question seems to be of general importance.

\section{Material and methods}

The study group comprised 24 patients (10 females and 14 males) with early diagnosed PD. The mean age of patients was $67 \pm 8.4$ years (ranging from 51 to 84 years). The duration of the disease ranged from 1 to 5 years. The patients have never before been treated with L-DOPA preparations. Peripheral blood was taken before the onset of treatment and after medication with L-DOPA compounds. The duration of treatment was $207 \pm 12$ days, ranging from 181 to 229 days. The patients were treated with 300 to $800 \mathrm{mg}$ of an L-DOPA compound per day. The following drugs were used: Madopar HBS (100 mg of L-DOPA) and Madopar 62.5 (50 mg of L-DOPA). The dosage was adjusted to the response in the course of hospital observation. From that time on, the patients were kept on a stable dose of L-DOPA. Some details of the applied treatment are presented in Table I.

The control group consisted of 29 persons (14 females and 15 males) aged from 46 to 86 years (mean $64.4 \pm 9.4$ years), without any evident disease of the central nervous system or extrapyramidal syndrome.

Lymphocyte subsets were analysed by flow cytometry with the aid of specific monoclonal antibodies purchased from the Becton Dickinson Company (San Jose, LA). The evaluation was performed using the Cytoson Absolute (Ortho Diagnostic System) at the wavelength of $480 \mathrm{~nm}$. The obtained relative results were analysed using the Immuno Count II program. The statistical analysis of results was performed using the Mann-Whitney $U$ test to determine significance of differences between initial values and those after L-DOPA treatment.

\section{Results}

The studied group of patients with PD demonstrated an increase in the CD95/CD3 lymphocyte subset, assumed to represent a marker of apoptotic cells, and an increase in the number of $\mathrm{Apo}(+) \mathrm{Pi}(+)$ positive cells, representing cells killed by apoptotic processes in their late phase (Table II).

After treatment with L-DOPA, the immunomarkers of peripheral blood cells demonstrated a relative increase in CD16 lymphocytes, while the percentage of CD19 lymphocytes, and the ratios of CD4/CD8 and CD95/CD3 were decreased (Table III).

The number of granulocytes dead due to the apoptotic process in its late phase significantly declined in treated patients (Table III).

\section{Discussion}

Immune abnormalities were documented in PD, including important occurrence of autoantibodies against selected neuronal structures with high numbers of activated microglial cells in the extrapyramidal system, predominantly in the substantia nigra. Activated microglia may participate in the death of neurons and reflect activity of toxic substances. Neuroinflammation in PD also seems to be connected with peripheral immune reactions. Changes in lymphocyte subsets in cerebrospinal fluid and in peripheral blood were also observed in PD, accompanied by enhanced synthesis of immunoglobulins and cytokines and augmented production of acute phase proteins. The alterations in mononuclear cell numbers in peripheral blood of PD patients have already been extensively discussed but have only partially been related to age-matched control groups. The later comparison is essential because of the senescence-accompanying increase in CD4(+) CD25(+) regulatory cells, noted in parallel to a general decline of immune responses. Hence, observations indicating that the involvement

Table I. Medication of Parkinson's patients with L-DOPA

\begin{tabular}{|lccccc|}
\hline $\begin{array}{l}\text { L-DOPA dose } \\
\text { in mg per day }\end{array}$ & 300 & 400 & 450 & 600 & 800 \\
\hline $\begin{array}{l}\text { Number } \\
\text { of patients }\end{array}$ & Total 1 & Total 2 & Total 3 & Total 13 & Total 5 \\
$(F-1, M-0)$ & $(F-0, M-2)$ & $(F-1, M-2)$ & $(F-6, M-7)$ & $(F-2, M-3)$ \\
\hline
\end{tabular}

F-female, $M$ - male

The L-DOPA compounds were: Madopar HBS - 1 tablet $100 \mathrm{mg}$ of L-DOPA and Madopar $62.5 \mathrm{mg}$ containing $50 \mathrm{mg}$ of L-DOPA+-- 


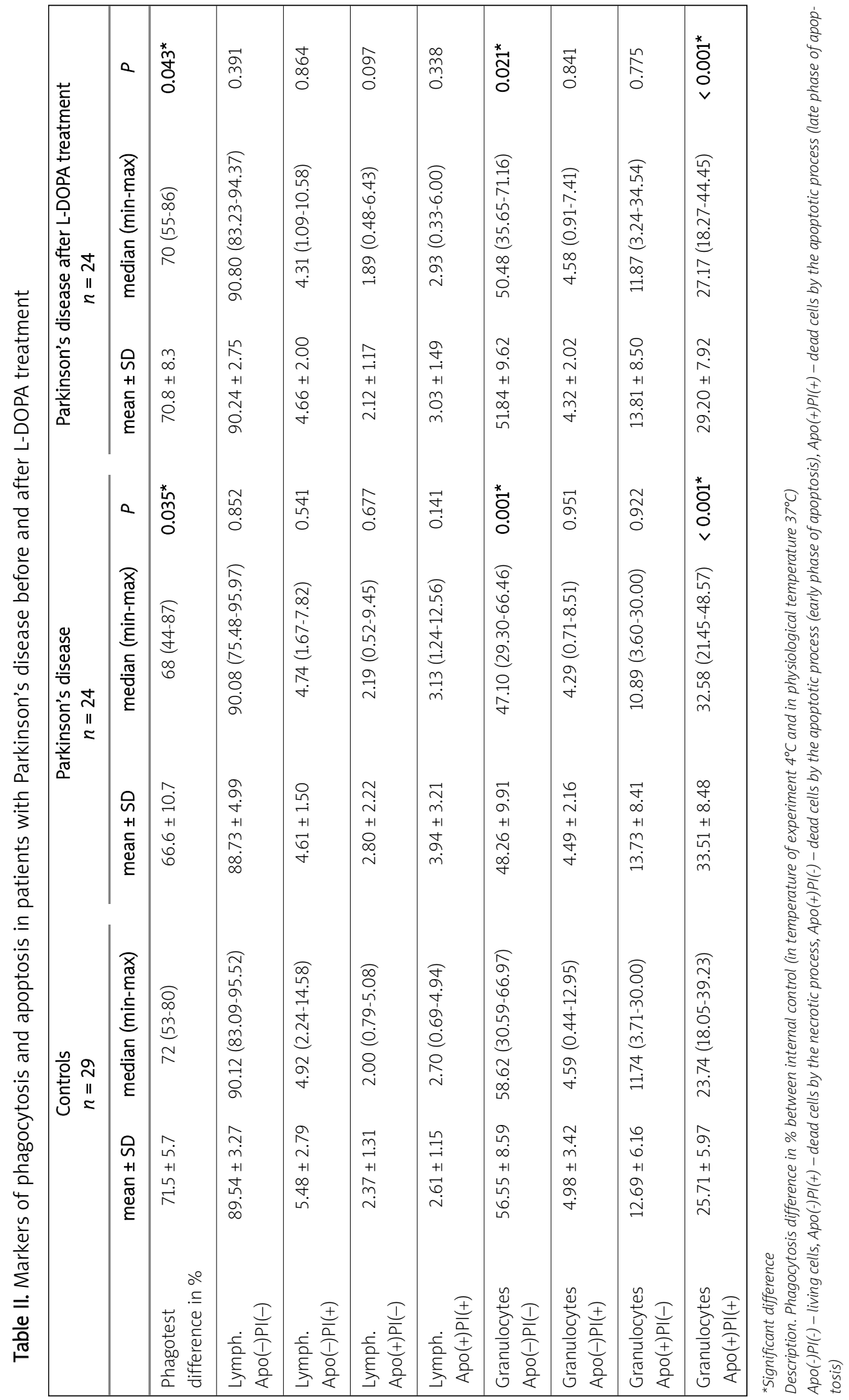




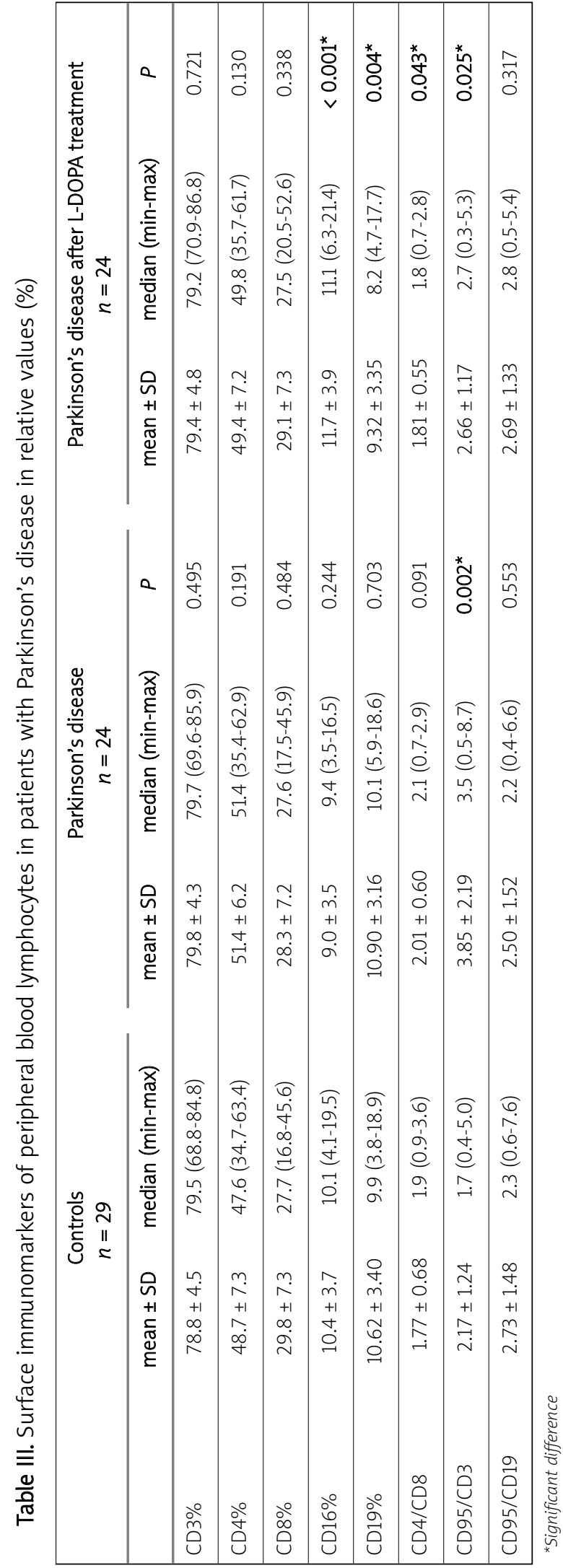

of T4+ helper/inducer/cells may be decreased in PD seem to be controversial.

The results of our studies do not demonstrate any significant differences in CD4, CD8, CD16 and CD19 counts in peripheral blood lymphocytes of PD patients, when matched with age appropriate controls. The same pertains also to the ratio of CD4/CD8 lymphocytes.

The results of our studies, demonstrating in PD a significant increase of lymphocyte subsets CD95/CD3, the markers of an antigen located on cells affected by apoptotic processes, can serve as a valid argument for the statement that dopaminergic cell death is influenced by an innate immune system, which has already been documented in the experimental mouse model of PD [2]. Our finding, demonstrating an increased number of granulocytes dead by apoptosis in its late phase, is consistent with the data.

Enhanced apoptosis in peripheral lymphocytes of PD patients was also shown by Calopa et al. [3]. According to the study, spontaneous and activation-induced apoptosis of CD4(+) T cell subsets was increased, thus pointing to enhanced susceptibility to apoptosis with Fas involvement. Invading $T$ lymphocytes contributing to neural cell death via the Fas/Fasl pathway have also been shown in the experimental mouse model of PD [2].

As to the effect of L-DOPA treatment on immunological processes in $\mathrm{PD}$, there is only very scanty information available. Fiszer et al. [6] reported that treatment with levodopa induced a selective effect on the immunological system of patients with PD as indicated by an increase in the synthesis of interleukin-1 and in IgM and IgA levels in blood plasma. Bas et al. [1] found that lymphocyte populations are not dependent on levodopa treatment in patients with PD.

In our study material we observed that after treatment with L-DOPA an appreciable increase had occurred in the relative amount of CD16 and CD19 lymphocytes, accompanied by a decrease in the CD4/CD8 ratio and in the number of lymphocytes carrying CD95/CD3 antigens. The CD95 antigen is characteristic of apoptotic cells within T/CD3(+) lymphocyte population. The observations are in line with our results, showing that after medication with levodopa the number of living Apo(-)PI(-) granulocytes had increased in parallel with a significant decrease in the number of dead granulocytes, resulting from an enhanced apoptotic process in the late phase of apoptosis.

Apoptosis-related proteins are elevated in the striatum of patients with PD, and this indicates that neuronal death in this disease involves an apoptotic 
process [10]. Indirect signs of apoptosis were observed in brain neurons and lymphocytes of peripheral blood. T cells manifested increased susceptibility to apoptosis with Fas involvement [3]. Therefore, our results confirming the impact of levodopa treatment on apoptotic processes in the peripheral blood of patients with PD supplement the understanding of L-DOPA's positive effect in PD patients.

\section{References}

1. Bas J, Calopa M, Mestre M, Mollevi DG, Cutillas B, Ambrosio S, Buendia E. Lymphocyte populations in Parkinson's disease and in rat models of parkinsonism. J Neuroimmunol 2001; 113: 146-152.

2. Brochard V, Combadière B, Prigent A, Laouar Y, Perrin A, BerayBerthat V, Bonduelle O, Alvarez-Fischer D, Callebert J, Launay JM, Duyckaerts C, Flavell RA, Hirsch EC, Hunot S. Infiltration of CD4+ lymphocytes into the brain contributes to neurodegeneration in a mouse model of Parkinson disease. J Clin Invest 2009; 119: 182-192.

3. Calopa M, Bas J, Callen A, Mestre M. Apoptosis of peripheral blood lymphocytes in Parkinson patients. Neurobiol Dis 2010; 38: 1-7.

4. Chung YC, Ko HW, Bok E, Park ES, Huh SH, Nam JH, Jin BK. The role of neuroinflammation on the pathogenesis of Parkinson's disease. BMB Rep 2010; 43: 225-232.

5. Członkowska A, Kurkowska-Jastrzębska I, Członkowski A, Peter D, Stefano GB. Immune processes in the pathogenesis of Parkinson's disease - a potential role for microglia and nitric oxide. Med Sci Monit 2002; 8: 165-177.

6. Fischer U, Piotrowska K, Korlak J, Członkowska A. The immunological status in Parkinson's disease. Med Lab Sci 1991; 48: 196 200.

7. Fischer U. Does Parkinson's disease have an immunological basis? The evidence and its therapeutic implications. BioDrugs 2001; 15 351-355.

8. Fischer U. Selected aspects of immunological disorders in Parkinson disease. Neurol Neurochir Pol 2004; 38: S63-66.

9. Hirsch EC, Hunot S, Hartmann A. Neuroinflammatory processes in Parkinson's disease. Parkinsonism Relat Disord 2005; 11 Suppl 1: S9-S15.

10. Jellinger KA. Cell death mechanism in Parkinson's disease. J Neural Transm 2000; 107: 1-29.

11. McGeer PL, Itagaki S, Akiyama H, McGeer EG. Rate of cell death in parkinsonism indicates active neuropathological process. Ann Neurol 1988; 24: 574-576.

12. Pfeiffer RF. Neuroinflammation and Parkinson disease: the silent battleground. Neurology 2009; 73: 1434-1435.

13. Rugbjerg K, Friis S, Ritz B, Schernhammer ES, Korbo L, Olsen JH. Autoimmune disease and risk for Parkinson disease. Neurology 2009; 73: 1462-1468.

14. Smith PF. Inflammation in Parkinson's disease: an update. Curr Opin Investig Drugs 2008; 9: 478-484. 